This item was submitted to Loughborough's Research Repository by the author.

Items in Figshare are protected by copyright, with all rights reserved, unless otherwise indicated.

\title{
Human perceptions of sports equipment under playing conditions
}

PLEASE CITE THE PUBLISHED VERSION

http://dx.doi.org/10.1080/026404101750238944

PUBLISHER

(C) Taylor \& Francis

VERSION

AM (Accepted Manuscript)

LICENCE

CC BY-NC-ND 4.0

REPOSITORY RECORD

Roberts, Jonathan R., Roy Jones, Chris G. Harwood, Sean R. Mitchell, and Steve Rothberg. 2019. "Human Perceptions of Sports Equipment Under Playing Conditions". figshare. https://hdl.handle.net/2134/10859. 
This item was submitted to Loughborough's Institutional Repository (https://dspace.lboro.ac.uk/) by the author and is made available under the following Creative Commons Licence conditions.

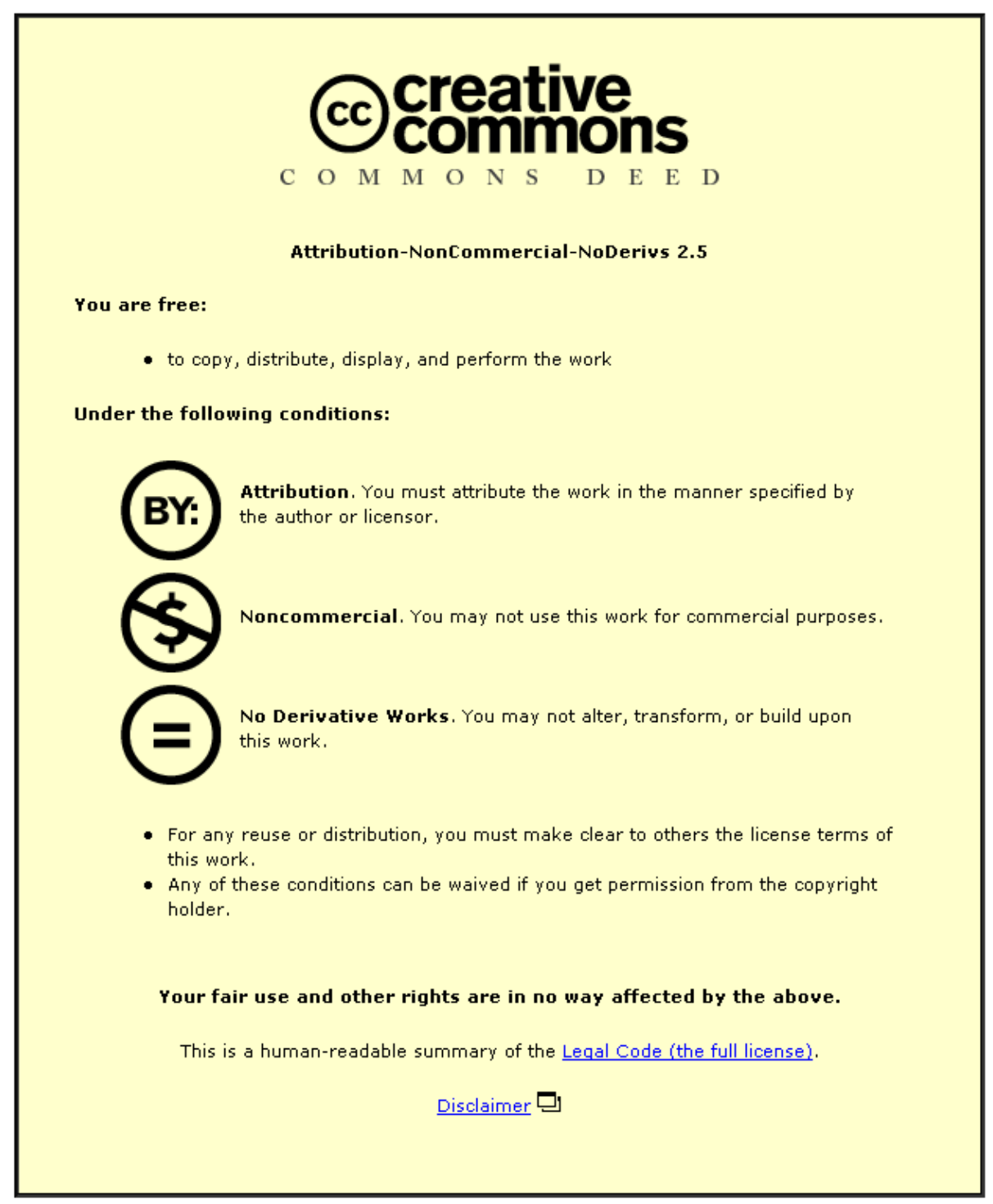

For the full text of this licence, please go to: http://creativecommons.org/licenses/by-nc-nd/2.5/ 


\title{
Human perceptions of sports equipment under playing conditions
}

\author{
J.R. Roberts ${ }^{1}$, R. Jones ${ }^{1}$, C. Harwood ${ }^{2}$, S. Mitchell ${ }^{1}$ and S.J. Rothberg ${ }^{1}$ \\ ${ }^{1}$ Wolfson School of Mechanical and Manufacturing Engineering, Loughborough University, Loughborough, LE11 $3 T U$. \\ ${ }^{2}$ Department of Physical Education, Sports Science and Recreation Management, Loughborough University, Lough- \\ borough, LE11 3TU.
}

\begin{abstract}
Assessment of sports equipment 'performance' is generally derived from physical and technical parameters, such as power, speed, distance and accuracy. However, from a psychological perspective, players need to feel comfortable with their equipment and confident in its properties. These factors can only be measured via the subjective assessment of individual perceptions. Using a study of a group of elite golfers as an example, this paper presents a formalised approach for eliciting and structuring player's descriptions of their perception of sports equipment. Qualitative methods of inquiry are employed to generate perceptions from a group of professional golfers $(n=15)$ during play testing. The equipment characteristics of significance to the golfers emerged from an inductive analysis of their responses. However, whilst this method of representation of the results was invaluable in identifying the key components or dimensions of a player's subjective perception, it was insufficient to describe the potential relationships between the dimensions. With this in mind, a new technique, entitled structured relationship modelling, was developed to illustrate these associations. Ten general dimensions emerged from the analysis, of which three are presented in this paper along with a section of the relationship model. These results demonstrate the effectiveness of qualitative techniques for eliciting human perceptions and of structured relationship models for representation of the associations found.
\end{abstract}

\section{Introduction}

In today's expanding sports equipment market, performers at all ability levels face an ever-increasing range of equipment from which to choose. Subjective assessment of equipment capability has been used for many years by manufacturers and players but relatively few studies have attempted to develop a systematic approach to the measurement of human perceptions (Hocknell et al., 1996; Merkel and Blough, 1999). The choice of equipment may often be based on the belief that performance enhancement will result from its use, with cost sometimes a secondary consideration especially for the more able performer. These enhancements may take the form of increased power, speed, distance or accuracy. However, such benefits may not be achieved if the player feels physically or psychologically uncomfortable using the equipment. The degree of personal comfort may be a key determinant when selecting sports products, yet a systematic approach for assessing such a factor remains insufficiently developed. As the major sector in the global sports equipment market, golf has been used for this study, which investigates how the characteristics of a golf shot affect a player's evaluation of the equipment used.

In golf, the player's perceptions of the shot result from the feedback received from a number of internal and external sources. Kinaesthetic perception describes the internal, proprioceptive feedback by which the golfer senses the sequence and nature of limb movements that form the swing (Sherwood, 1993). By comparing this with the memory of previous swing experiences, the golfer judges the quality of that swing. The player's perception of the shot will also be affected by external sources that either induce somesthetic sensations in the hands or provide exteroceptive information to the eyes and ears (Schmidt, 1991; Sherwood, 1993). Even before a shot has been played, the golfer will begin to receive salient feedback from observing and holding or waggling the club. Additional feedback will also be received during the swing, from impact and from post-impact sensation (Cochran and Stobbs, 1968; Hedrick and Twigg, 1994; Hocknell et al., 1996).

Perception can be defined as "our conscious interpretation of the external world as created by the brain from a pattern of nerve impulses delivered to it from sensory receptors" (Sherwood, 1993, p151). However, different people will not necessarily perceive the same sensory input in the same manner. Take, for example, Figure 1, in which, from identical visual input, it is possible to 'see' two faces in profile or a wineglass. This much used optical illusion illustrates how the brain interprets information received according to its own rules and it is important, therefore, to consider a number of significant points when analysing the player's subjective perceptions of golf equipment. Through differences in swing dynamics, sensory feedback may vary between different golfers using the same equipment. In addition, different golfers can receive the same feedback but interpret it and describe it differently. 


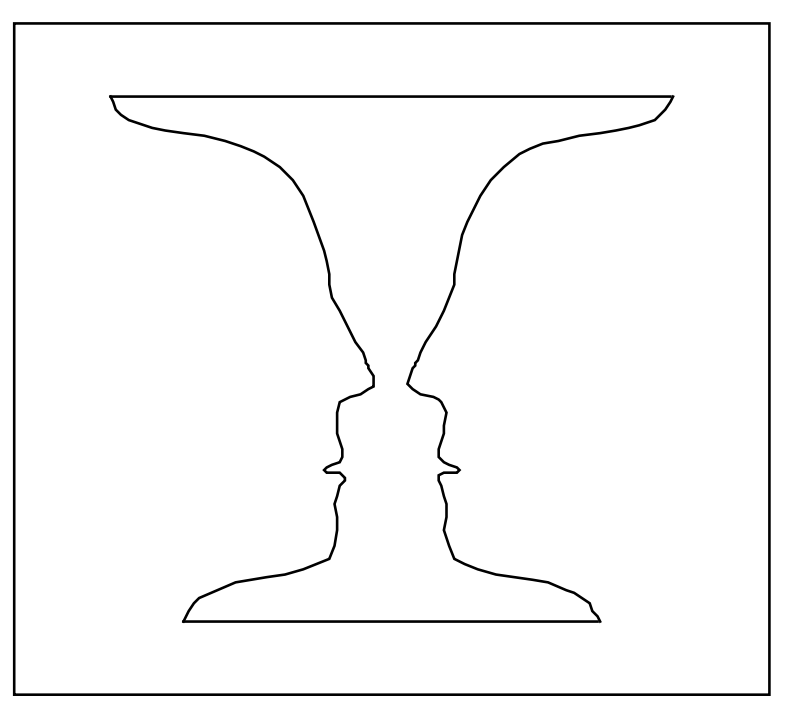

Figure 1 - Variable perceptions from the same visual input

Four previous studies (Hedrick and Twigg; 1994; Varoto and McConnell, 1995; Hocknell et al., 1996; Merkel and Blough, 1999) have investigated the effect of sound and vibration on the ill-defined concept of 'feel' of a golf shot. In most of these studies, however, only limited attempts were made to assess the golfers' subjective perceptions. Hedrick and Twigg (1994) compared vibration measurements taken from the golf shaft after centre, heel and toe impacts whilst Varoto and McConnell (1995) investigated the effect of a golf club's modal characteristics on the feel of a shot. However, neither study attempted to assess and therefore link these measurements to the perceptions of a golfer using the clubs. Merkel and Blough (1999) measured club vibration induced by the impact as well as conducting modal tests on a number of clubs. This data was then correlated with the player's subjective rating of the shot played - 'good' or 'bad'. "The subjective qualifications for a 'good' (or bad) hit included both feel and flight of the ball" (Merkel and Blough, 1999, p 515). Although the golfers' perceptions were included in this study, other researchers have demonstrated that a player's assessment of equipment is much more complex. In a preliminary study for this work, Hocknell et al. (1996) measured both the sound and vibration from each impact during a series of player tests. The golfer ranked each club-ball combination in terms of the feel of the shot played, from hard to soft, and the sound of impact, from high pitched to low pitched. A postal questionnaire was also used to obtain both quantitative and qualitative data from a larger sample of low handicap golfers on their perceptions of feel. The study by Hocknell et al. (1996) highlighted the requirement for an in-depth investigation into the characteristics that contribute to a player's complete perception of a golf shot. This study sets out to address this need.

To understand the characteristics that contribute to a golfer's perception of the equipment used, suitable research techniques are required for eliciting and analysing these components. A number of previous studies in sports psychology have employed qualitative techniques to ob- tain and analyse rich, detailed, descriptive data. For example, Scanlan et al. (1989a,b) developed a methodology that enabled acquisition and structuring of qualitative data on sources of enjoyment and stress for elite figure skaters. Open-ended questions were used during a series of in-depth interviews to collect data from a sample of skating coaches. An inductive analysis was then performed to structure the data, which allows the significant components to emerge from the data through a process of clustering together common themes. "Clustering involves comparing and contrasting each quote with all the other quotes and emergent themes to unite quotes with similar meaning and to separate quotes with different meanings" (Scanlan et al., 1989b, p 68). The process is then repeated with the base level themes identified being compared and contrasted in a similar manner generating higher level themes. At each level of analysis, an individual theme should be inclusive, in that it adequately captures the clustering of lower order themes that comprise it, and all themes should be mutually exclusive, with clear distinctions between them (Scanlan et al., 1989b). "The analysis continues building upward until it is not possible to locate further underlying uniformities to create a higher theme level" (Scanlan et al., 1989b, p 68). The highest level theme is referred to as a 'general dimension'. Other studies have used similar methods to elicit information from Olympic wrestlers (Gould et al., 1992a,b), junior tennis players (Harwood, 1997) and swimmers (Hanton and Jones, 1999).

To develop an understanding of the perceptions, feelings, thoughts and knowledge of a golfer, it is essential that minimal constraint be placed upon the responses of the player. It is important that the player is allowed the "opportunity to express himself about matters of central significance to him rather than those presumed to be important by the interviewer. That is, ... it uncovers what is on the subject's mind rather than his opinion of what is on the interviewer's mind" (Merton and Kendall, 1946, p 545). The use of open-ended questions during semistructured interviews allows the participants the freedom to respond in their own terms and phrases about issues of importance to them. A subsequent inductive analysis of these responses enables the significant issues to emerge without presupposing in advance what those important dimensions will be (Patton, 1990). Thus, the template of semi-structured interviews followed by an inductive content analysis was deemed ideal for this study. However, it became apparent during the analysis procedure that although this method was invaluable in identifying the key components of a player's subjective perception, it did not enable any interactivity between dimensions to be investigated. As a result an additional analysis stage was introduced to facilitate exploration of inter-dimension relationships and to develop a more suitable method for representing the findings, and this will be discussed later. 


\section{Method}

\section{Participant Selection Criteria}

For this study, it was specified that the population sample consisted of male professional golfers aged between 25 and 55 years old. Elite players were chosen because it is believed that a golfer's sensitivity to differences in equipment characteristics increases as the player improves and gains experience. The age range of 25 to 55 years is thought to represent the period when a golfer will still be competing and will have sufficient experience. The wide age range allows any differences between different generations of golfers to be assimilated. Exclusively male golfers were chosen since elite male golfers were more readily available. However, the same procedure can easily be replicated in future studies to investigate population samples of other groups of golfers.

A minimum sample size of fifteen was initially selected based on experience of a number of previous research studies involving interviews with elite performers (Scanlan et al., 1989a,b; Gould et al., 1992a,b; Harwood, 1997; Hanton and Jones, 1999). A sample size of fifteen was also considered to provide a balance between breadth and depth of knowledge obtained.

\section{Participants}

Professional golfers satisfying the selection criteria were identified based upon their position in the 1997 UK PGA Midlands Order of Merit Table. Of those approached only two refused, one because of illness and the other due to a commercial conflict of interest. The sample of golfers had a mean age of 38, $\sigma=10$ years, and had been playing at a professional level for a mean of $19, \sigma=11$ years. After interviewing fifteen players, it became apparent that a 'saturation' stage had been reached where no new information appeared to be emerging and so the interview program was terminated (Biddle et al., 2000).

\section{Instrumentation}

The procedure followed for the design of this study is illustrated in Figure 2. The flow diagram was used to aid the design of the research study by providing a logical progression through the major design issues involved in the process. The design decisions taken and the compilation of an interview guide are discussed in the following section.

A naturalistic style of inquiry was deemed more suitable for this study as it is a "discovery-oriented" approach with minimal investigator manipulation of the study setting and no prior constraints placed on the outcomes of the research (Guba, 1978; Patton, 1990). By utilising this type of inquiry, the characteristics of importance to the golfer could be investigated, as little restraint would be imposed on the responses of the golfer by the study design.

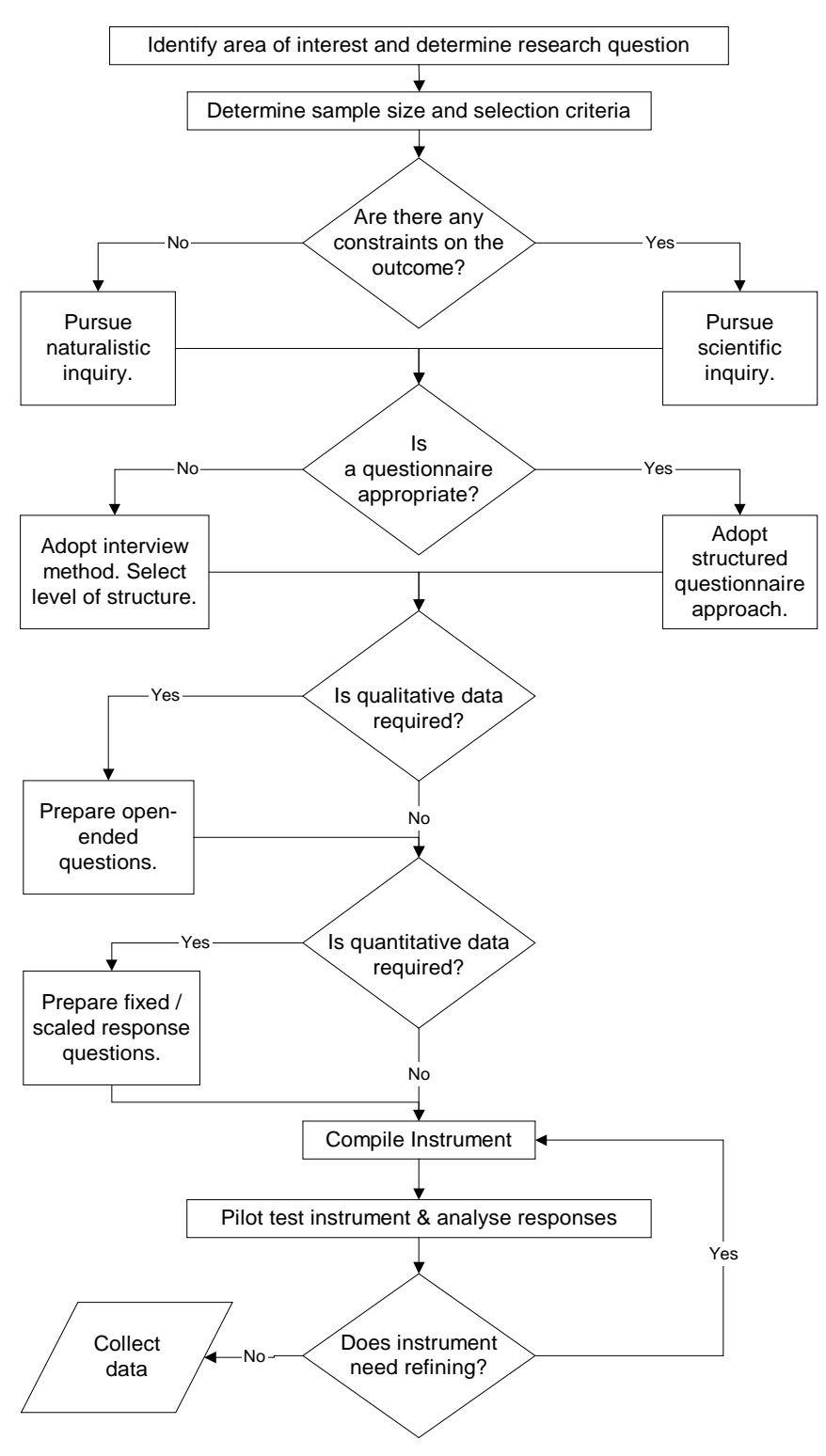

Figure 2 - Study design process

To collect data, the interview technique was considered more appropriate for this part of the study than the questionnaire. Depending on the level of structure, an interview can offer increased flexibility to vary the content, sequence and wording of questions and can provide greater opportunities for probing. These features allow responses to be explored and result in completeness of information and depth of understanding. An interview guide was developed that outlined the subject areas to be explored giving continuity to the interview and comparability between interviews.

\section{Interview Guide and Schedule}

The interview guide developed had a basic structure comprising several sections, with the same sequence of sections used for each interview. Within each section, an introductory question was posed with some possibilities for further questioning and discussion listed. After the initial question had been asked, the interviewer had complete freedom and flexibility to explore and probe the 
golfers' responses. However, topics were only discussed if they had been introduced into the discussion by the golfer. Open-ended questions were used to obtain qualitative data in the form of detailed descriptions containing the respondents' own terms and phrases. "Open-ended situations can also result in unexpected or unanticipated answers, which may suggest hitherto unthought-of relationships or hypotheses" (Cohen and Manion, 1980, p 313).

The first stage of each interview was conducted outdoors, on the driving range of the golf club where the Professional was resident, thus placing him in surroundings in which he would be comfortable and familiar. The golfer was then asked to hit several shots with each driver club and ball and once he had formulated an opinion, describe his perception of that combination. Verbal probes were then used to discuss his perceptions in more detail. Clubheads constructed from titanium, stainless steel, persimmon, laminated wood and graphite were assembled with steel and graphite shafts of different flexibilities. An assortment of grip styles was also used and two different constructions of golf ball, a two-piece, surlyn ball and a three-piece, wound, balata ball, were provided. The various combinations of club construction and ball type were thought to encompass the spectrum of feel characteristics associated with golf equipment. The golfer was introduced to this section of the interview with the following explanation.

First of all I would like you to hit a number of golf shots with each club/ball combination. After you have played each shot I want you to describe your perception of 'feel' of that shot. If you wish to hit a number of shots to get used to the club before you respond, please do. It is important that you comment upon the 'feel' of the shot you have played and avoid allowing preconceptions of different club/ball designs to affect your responses.

Examples of specific questions posed to encourage player responses included "How do the traditional wooden clubs 'feel' in comparison to the modern titanium clubs?" and "How does the 'feel' of the shot differ between the two different ball types?" Terms and phrases used by the golfer to describe his perceptions were noted whilst clarification and elaboration probes were used to obtain detailed descriptions and a more comprehensive understanding (Patton, 1990). As these types of question are specific to a particular characteristic they were only used once the golfer had introduced that characteristic into the discussion. Typical probes used included "What do you mean by 'solid feel'?", "What don't you like about the appearance of that club?" and "How would you describe the sound from that club?"

The first stage of the interview served two purposes. It placed the golfer in an environment in which he was comfortable and at ease but, most importantly, it stimulated the player's mind, increasing the quantity and quality of responses. Golfers were more able to talk in depth about their perceptions as they used each club and ball than when they were required to recall past experiences of their perceptions.
The second stage of the interview did not require the golfer to hit shots. The remaining sections were used to ascertain the reasons why the golfer selected his current set of equipment, the characteristics of an 'ideal' golf shot and the relative importance of the characteristics discussed during the interview. In the final section the golfer was asked to provide some feedback on the interview itself and to suggest possible improvements that could be made.

Five pilot interviews were carried out to determine the suitability of the interview technique and a number of modifications were made to remove redundant sections and reword ambiguous questions.

\section{Recording System}

Each interview was recorded to enable complete and accurate information to be logged in an efficient manner. As part of the interview took place outdoors in varying weather conditions with the golfer hitting a number of shots, a specialised recording system had to be developed that fulfilled several criteria.

- Every spoken word by each individual must be clearly and audibly recorded on a single medium to allow complete and accurate transcriptions to be produced with a minimum of effort.

- Recording equipment must be unobtrusive and not impede the golfer's swing.

- Recording equipment must be portable, battery powered and quick to set up.

A dual, wireless, lapel microphone system feeding into a radio transmitter satisfied these requirements. In the interview situation, participant and interviewer can talk at the same time making transcription difficult, so a minidisc stereo recording device was used which allowed information from each microphone to be recorded on separate tracks.

\section{Data Analysis}

The organisation of raw data into structured, meaningful themes can be approached from two perspectives. A deductive analysis involves arranging quotes into a set of pre-determined categories while an inductive analysis allows the themes and categories to emerge from the quotes. Previous studies in sports psychology (Scanlan, et al., 1989a,b; Gould et al., 1992a,b; Harwood, 1997; Hanton and Jones, 1999) have argued that they followed an inductive process for structuring qualitative data. To allow those characteristics important to the golfer to emerge and to minimise restrictions imposed by the investigator, an inductive analysis was viewed to be the most suitable approach for this study.

The software package QSR NUD*IST was used during the analysis to handle the unstructured, qualitative data obtained. NUD*IST assists in shaping understanding of the information by enabling categories of data to be created and emergent categories to be linked. Exploration tools are also provided to form and test theories grounded in the data. 


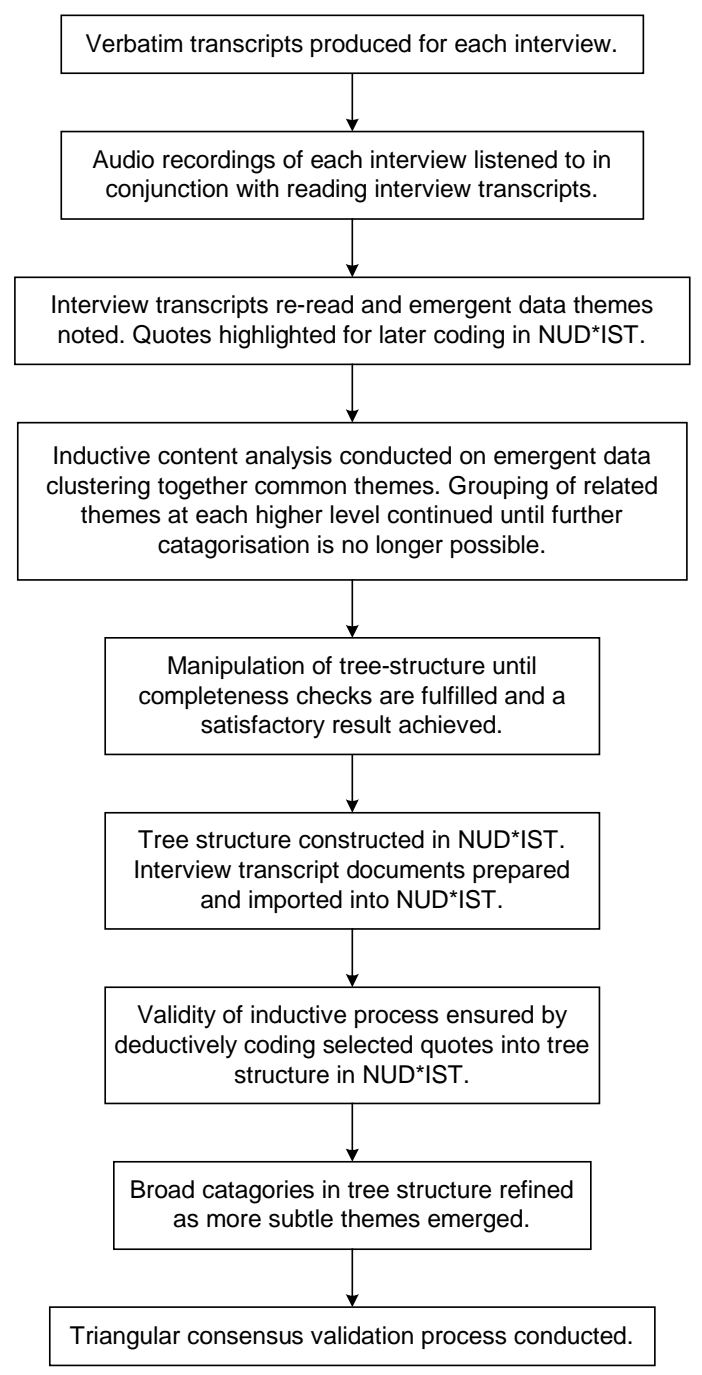

Figure 3-Schematic of the data analysis procedure

The inductive analysis procedure used, as illustrated in Figure 3, began with the verbatim transcription of each interview. Familiarisation with the data involved listening to the interviews, reading each transcript several times, highlighting important quotations and making notes on the subject matter discussed during each interview. The selected quotes became the basic unit of analysis.

In the next stage, the inductive analysis advocated by Scanlan et al. (1989a,b) was conducted. A number of guidelines are suggested by different authors to assist in the process of structuring and making sense of large volumes of qualitative data. Patton (1990) advocates focusing the analysis by considering the purpose of the study and attempting to answer the research question formulated at the start of the study design process.

One issue that often arises during an inductive analysis is the problem of alternative classification schemes. Take for example four of the base level themes, 'Feel Club Twist from Off-Centre Shots', 'Hard Feeling Ball', 'Soft Feeling Ball' and 'Hard Feel from Off-Centre Shots' that emerged during the analysis of golfer's responses. As illustrated in Figure 4, there are a number of different ways of grouping the themes at a higher level. In this situation, Patton (1990) suggests developing each classification system and then establishing priorities to determine which of the category systems is more important than the others. A technique used during this study to eliminate different systems and refine the configuration was to consider each dimension in terms of redundancy. A dimension can be considered to be redundant if all the base level themes can successfully be re-coded into another dimension. If a large number of unassignable or overlapping themes still exist, this is evidence of a basic fault in the category system (Guba, 1978).

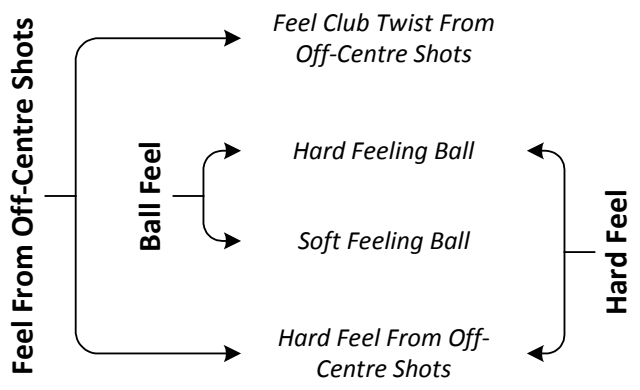

Figure 4 - Alternative classification schemes

Finally the structure was tested for completeness using a number of checks.

- The analysis should leave as few unassignable themes as possible. Any remaining unclustered themes should be either disregarded if indistinguishable or retained if important (Scanlan et al., 1989b).

- The structure should be reasonably inclusive of the data and information that exists. If the set of categories does not appear to be sufficient, on logical grounds, to cover the facets of the problem, the structure is probably incomplete (Guba, 1978).

The structure for each dimension was then created in NUD*IST, and a deductive process of coding the previously highlighted quotes into the composed structure was used to validate the inductive process. With the data in a more organised format more subtle themes emerged allowing the creation of refined themes from initial base level categories.

Triangular consensus validation was then used to minimise any bias from the principal investigator and this is discussed further in the following section.

\section{Quality of Results}

A number of steps were taken to obtain trustworthy results by minimising individual bias. Firstly, two investigators conducted each interview to reduce potential errors that can arise from a single person collecting all the data (Patton, 1990). Possible sources of error in the interview technique (Cohen \& Manion, 1980) include:

- The attitudes and opinions of the interviewer

- A tendency for the interviewer to see the respondent in his own image

- A tendency for the interviewer to seek answers that support preconceived notions

Journal of Sports Sciences, 2001, 19, 485-497 
- The interviewer misinterpreting the responses

- The respondent misunderstanding what is being asked

During the interviews, these sources of error were minimised where possible by using unambiguous questions that did not guide or force responses in a particular manner. The two interviewers received methodological guidance on interview techniques from an experienced colleague and through reading relevant texts. Five pilot interviews were conducted prior to the main interview programme to refine techniques and become familiar with the terminology used by the golfers.

Secondly, triangular consensus validation (Scanlan et al., 1989b, Patton, 1990) was conducted, during which the emergent dimensions were discussed by the two investigators present at each interview, together with a third person also experienced in qualitative data analysis, until agreement was reached at each stage of the analysis.

Upon completion of the analysis, five further interviews were conducted with a sample of elite American golfers. Similar themes were discussed, albeit using alternative, American terminology, which could easily be coded deductively into the structure created from UK golfers' responses. For example, both groups of golfers discussed the relative stiffness of a shaft, but American golfers would use the terms 'unstable' or 'stable' as opposed to the British golfers who used the corresponding terms 'whippy' and 'firm'. Although it was encouraging that no additional themes emerged, a study with a larger sample of American golfers would be required to confirm that the results of this study are applicable to a similar group of American golfers.

\section{Results}

The purpose of the study was to identify the important characteristics that affect a player's perception of the equipment used when hitting golf shots and, in so doing, to develop a methodology that could be used for the assessment of a wide range of sports equipment.

Ten general dimensions emerged from the inductive analysis of the golfers' responses. However, the complete set of results is too extensive to report within this paper and so the following three dimensions have been selected, based upon their interactivity, for presentation: 'Shaft Feel', 'Club Weight' and 'Club Control'.

The tree-structures for these dimensions are illustrated in Figures 5, $6 \&$ \% . Each structure illustrates how the analysis progressed from the initial quotes in the lefthand column, through each different level of clustering to the general dimension at the right-hand side.

Golfers' perceptions associated with shaft flexibility and shaft length are grouped together in the general dimension 'Shaft Feel', as shown in Figure 5. 'Shaft Flexibility' is a high-order category that contains themes covering the golfers' perception of the shaft flexing when the club is either waggled or swung. The quotes are grouped into categories depending on whether the shaft was perceived by the golfer to be 'too flexible', 'well suited' or 'stiff'.
By far the most popular way of describing a shaft that felt too flexible was with the term 'whippy'.

The shaft is totally unsuited to me I should think, it feels very whippy, not very strong at all.

At the other end of the flexibility scale the term 'stiff' was used.

That felt very stiff in the shaft... the others felt like the head was coming in too quickly, like the head was wanting to come in before the shaft. This one felt the opposite, this felt like the head was not wanting to strike the ball at all.

Shafts that golfers considered were suitable for their swings were described in a variety of ways and so were grouped together in a general category entitled 'Well Suited / Firm / Together Feeling Shafts'.

It feels lovely... as soon as you pick a club up, you only have to have a couple of swishes with it... you feel as though you're going to be able to hit it. You know the composition's right from gripping the grip, having a couple of swings, you can feel the shaft, you can feel the head, you feel when the shaft is going to kick in and as soon as you pick a club up it's very evident you're going to be able to use it.

A separate category was created for the golfers who uniquely used the term 'solid' with reference to a shaft flexibility they approved of.

I felt the shaft was solid and there wasn't a lot of sort of kick in the shaft and it felt quite good really...

Two separate themes were created to accommodate the specific feel of the shaft twisting. General quotes were grouped together in the category 'Twisting Feel in the Shaft', whereas quotes that attributed the twist to an offcentre impact were collected together in the theme 'Feel Club Twist From Off-Centre Shots'.

I hit the last one a bit out of the toe and I felt the shaft twisting.

A number of golfers also described feeling the shaft 'release' or 'kick' prior to impact as the shaft recovered from the loading during the downswing. However, this was only considered desirable if the shaft released in time with the golfer's swing.

The shaft for me was probably just a little bit too stiff. I felt as though there was no release. When I released, the club wasn't releasing with me."

'Feel of Shaft Length' encompasses the quotes from golfers who perceived a difference in the lengths of the shafts.

"...with the difference in the length as well, it makes it far, far easier to generate or to create the feeling of width which then generates the more power.

The dimension 'Club Weight' encompasses the golfers' perception of differences in both overall weight and swingweight and is therefore subsectioned into these two themes, as shown in Figure 6. Swingweight is a measure of the weight distribution of a golf club about a fulcrum point usually 14 inches from the grip end of the club and is considered as a measure of the ease with which a club can be swung. Clubs with a large proportion of their mass distributed towards the clubhead have a heavy swingweight and therefore require more effort to swing. 


\section{Example Quotes}

Shaft's whippy for me, there's a lot more flex there.

The shaft feels quite whippy.

Shafts a bit whippy...which means you can't really hit at it you just have to wait for it...

You can almost feel that the shaft is almost too flexible.

The shaft feels very flexible.

That shaft is horrific. That is so flexible, it feels so soft.

I feel the shaft really twist and turn.

A lot of twist and turn in the shaft, you feel the head trying to twist. I mean I've hit that pretty much out of the middle and still the head was almost trying to twist and turn.

...again I felt the shaft giving too much, very flexible shaft.

...there's a lot more flex and give in the shaft...

I felt there was a bit of give in the shaft.

The shaft felt better in that one though, it felt a little bit more together, a bit firmer, it didn't feel as though it was twisting quite so much.

It feels like the whole thing is together.

That's the first one I've hit where the shaft flex feels the right flex for my swing.

Better shaft in this one, it's a lot more solid.

... the shaft felt great in that it felt... solid.

I think this one [Great Big Bertha] and the Titleist, the shafts have felt the best in these two.

You know, they feel as if they're really solid to be honest with you.

It felt too stiff that did

The shaft for me was probably just a little bit too stiff. I felt as though there was no release. When I released, the club wasn't releasing with me.

That felt very stiff in the shaft..

...you can feel the shaft want to kick a lot more at the bottom.

Some of these steel shafted clubs I could feel a real kick through the shot so that they felt a lot more solid throughout the swing.

I could feel the kick there, which I quite like.

... that was out of the heel, that one. When you mis-strike it you can really feel the club face twisting and turning.

I can feel when I hit it out of the toe that the club tries to twist open. And you can certainly feel when you hit it out of the heel that the club tries to twist closed.

...it did feel a bit longer than the Callaway.

Length of shaft has... a big part in my feel, and because I think it's the height of me, the longer the shaft, I don't get a good feel from... I don't feel comfortable.

Figure 5 - The general dimension 'Shaft Feel'

Low Order

Sub- Themes

High Order

Sub- Themes

7
Themes

Feeling Shafts

-

Flexible

Feeling Shafts

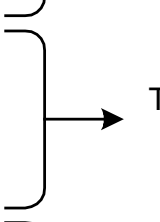

Twisting Feel

in the Shaft

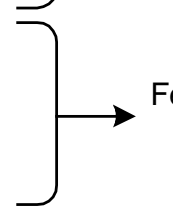

Feeling of Give

in the Shaft

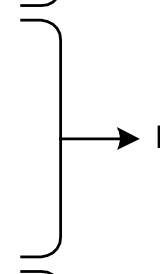

Well Suited /

Firm / Together

Feeling Shafts

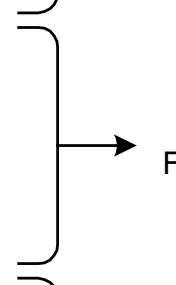

Solid

Feeling Shaft

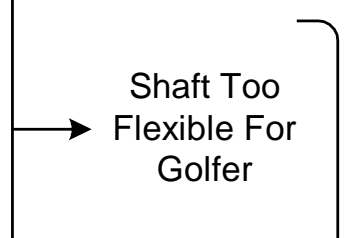

$\int$

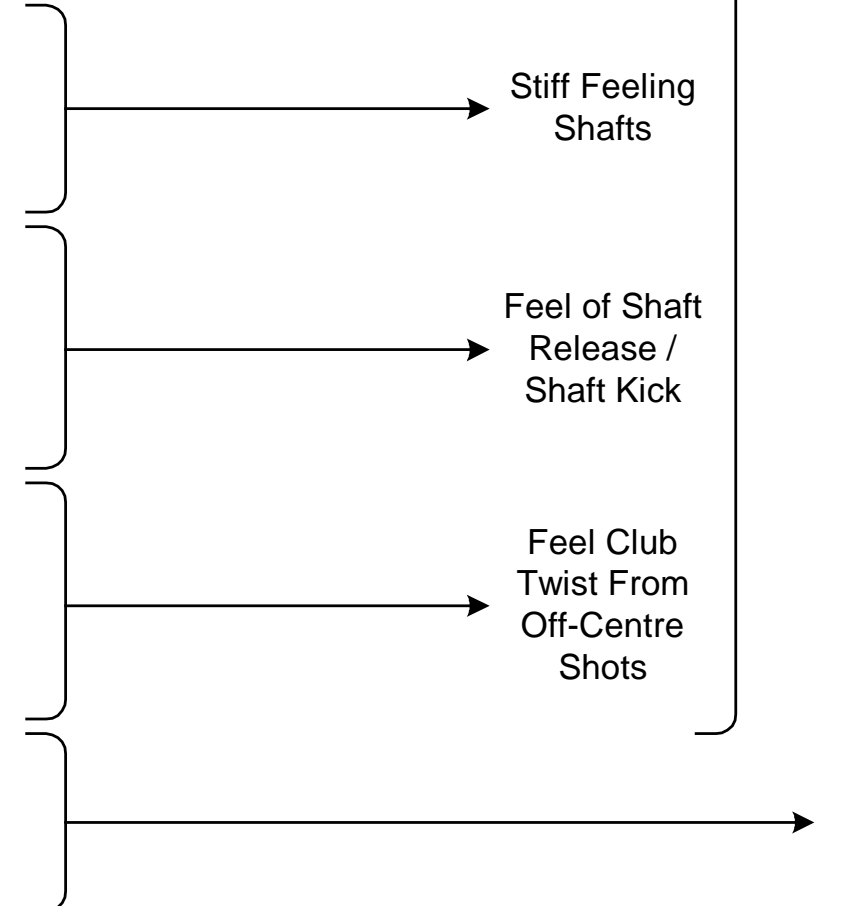

Shaft Flexibility

Feel

of Shaft

Length

Shaft Feel 


\section{Example Quotes}

...it feels quite cumbersome... it feels very heavy in the head.

The Titleist one definitely feels more head heavy.

The shaft certainly feels a lot heavier.

It feels the best balanced of the lot this one, so shaft weight and head weight for me is about spot on there.

You want the whole thing balanced together so that when you hit it, it feels it's all part of you.

You want the club to feel part of yourself really.

The balance of the club feels better.

A massive lighter swing weight initially, just through feel of swing.

...if I use the RCH 36 shaft, the head feels too light.

Now, this shaft feels very light.

...gone back to the Titleist now. Feels quite a bit heavier than the Callaway.

First impressions... when I put it down was that it was far too heavy. It felt really heavy and felt really heavy when I was swinging it as well..

That feels more clumsy as in overall weight is heavier than the persimmon.

...the club just feels a little bit light over all. A little bit feathery.

This one probably felt a little bit light.

Instantly feels quite a lot lighter.
Base Level

Themes

High Order

Sub- Themes

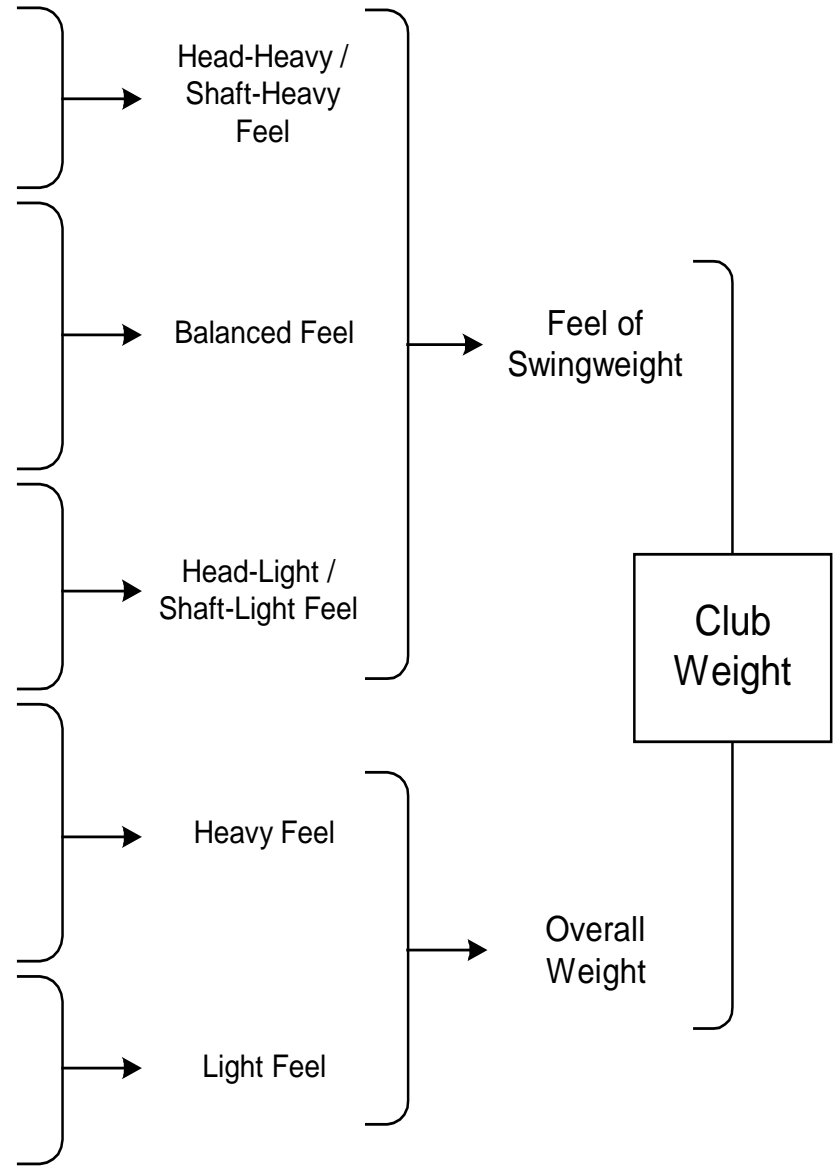

Figure 6 - General Dimension 'Club Weight'

\section{Example Quotes}

The ordinary wood...has the ultimate feel, it feels like it's a golf club that you're very much in control of, rather than it's in control of you.

The whole club swung very well, it felt nice. You felt as if you were in control.

...just feel as though I'm in control of the club head right throughout the shot.

I feel I've no control over that head at all. I don't know where it is.

This feels much more difficult to control...

... but I could not control it because of the length and the flex of the shaft.
Base Level

Themes

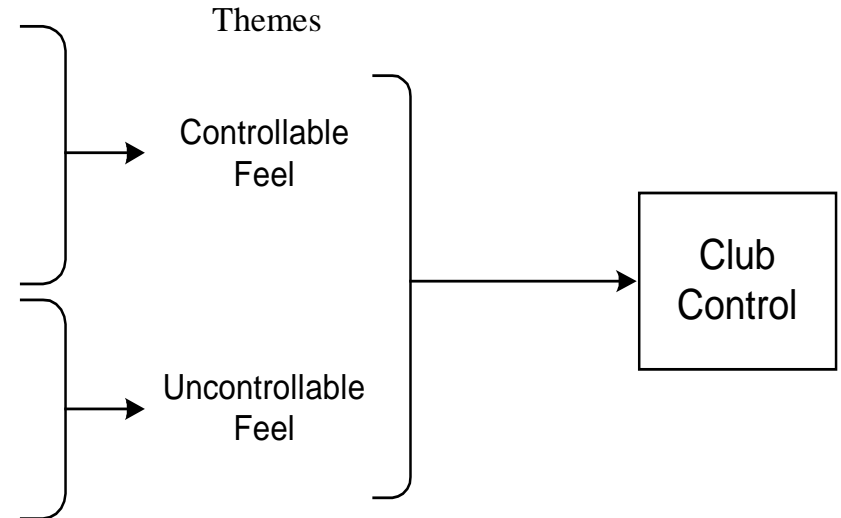

Figure 7 - General Dimension 'Club Control' 
Three themes emerged from the quotes describing the golfers' perceptions of different swingweight. These three categories effectively divide the swingweight scale into three sections. Clubs perceived as having a heavy swingweight were described as being 'head heavy' or 'shaft heavy'.

...from the top of the back swing when you change direction, if it's very head heavy you just feel like the head's going to be thrown out and you're going to hit it very early and hook the pants off it.

At the other end of the scale, clubs perceived as having a light swingweight were described as being 'head light' or 'shaft light'.

Just the shaft, it just feels so light the head and everything, I can't feel where it is through the swing.

Clubs in between were described as being 'balanced'.

It feels a nice club, it just feels very well balanced, as in the head and the shaft feel matched up...

'Overall weight' covers the themes of the club having an overall 'Heavy Feel' or an overall 'Light Feel'.

Oh, dear. This feels very weighty, extremely weighty. It is a... let the club work for you sort of club.

... it feels overall lighter, so it feels if I wanted to really lash one I could hit it and I'd feel I'm swinging the club, much more in control of the club... the club's not wanting to overtake me.

The dimension 'Club Control' brings together the contrasting themes of the golfers' feelings of being either in control or not in control of the club and is illustrated in Figure 7.

It's when you hit the golf ball, if you don't feel the club has swung you, if you feel you have control of the club, and the ball seems to come off with a proper spin on it, you're happy.

It's just because it's steel, I think, you feel as though you've lost a little bit of control there. It feels as if there's going to be a little bit more variation in the flight with a steel shaft.

\section{Analysis Limitations}

During this initial stage of the analysis, quotes were considered on the individual characteristics of the equipment or shot that were perceived by the golfer. To maintain the meaning of the quote descriptions were kept whole. This often resulted in quotes containing a number of themes, which had to be coded into numerous base level categories. Take, for example, the following quotation:

... the shaft is so flexible, I can feel that really flexing and twisting and turning, and for me, I just could not control it...

The golfer quoted above described three different perceptions, the shaft felt flexible, the shaft felt as though it twisted and the club felt uncontrollable. Initially, the quote was coded into the base level themes 'Flexible Feeling Shafts', 'Twisting Feel in the Shaft' and 'Uncontrollable Feel'. However, the quote also suggests that there is a relationship between the flexibility of the shaft and the degree of control the golfer has over the club. Further analysis of the data revealed numerous other interdimension relationships and it became apparent that the complete analysis could no longer be represented by simple tree-structures.

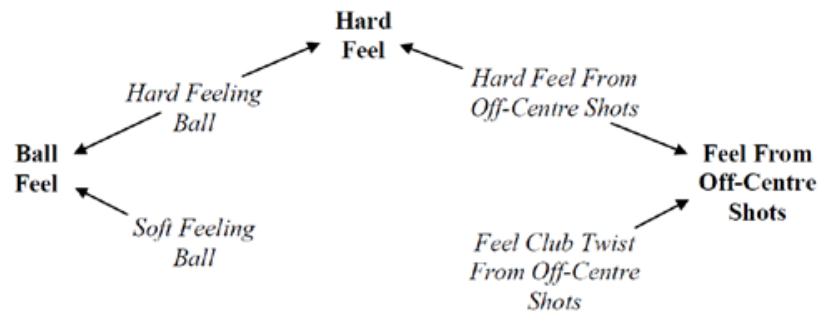

Figure 8 - Relationship map

A relationship 'map' was initially created during the analysis to represent the alternative classification schemes, as shown in Figure 4, which could be used to categorise the data. Using the same themes as an example, Figure 8 illustrates the way in which a map can be used to represent dimensions with shared themes. Each dimension was then assessed in terms of redundancy and any remaining shared themes then became interdimension relationships. In the example illustrated in Figure 8, 'Feel From Off-Centre Shots' proved to be a redundant dimension as all the base level themes were linked to other dimensions and therefore could be recoded into them. Completeness checks were performed on the structure and a diagrammatic style developed to visually distinguish between each theme level and the interdimension relationships.

\section{Structured Relationship Modelling}

A section of the structured relationship model that was developed from the initial map is contained in Figure 9, illustrating the dimensions discussed in the previous section and the relationships between them. The complete model, containing all ten general dimensions, shows thirteen inter-dimension relationships.

In Figure 9, two features of the shaft, flexibility and length, appear to affect the golfers' ability to control the club. The effect of shaft flexibility on the feeling of control was described by golfers particularly when the shafts were too flexible for them to control during their swing.

It doesn't feel like I've got as much control through the swing because the shaft is bending more than I'd like it to.

Shaft-wise it didn't feel very controllable. The shaft feels quite whippy.

Only one golfer made reference to the length of the shaft and its effect on control, believing he was more able to control clubs with shorter shafts.

It feels a lot shorter, (I'm) feeling more in control of this club because it's a lot shorter in the shaft.

The effect of the weight of the club on the feel of control over the club was a connection made by a number of the golfers interviewed. In general golfers feel less in control of heavier clubs and more in control of lighter clubs.

Because the club head's a lot lighter with that one, you can actually feel you've got more control at the end.

It certainly felt so much heavier to me that... I wouldn't like to play with it at all. I'd feel it was controlling me and not me controlling it. 


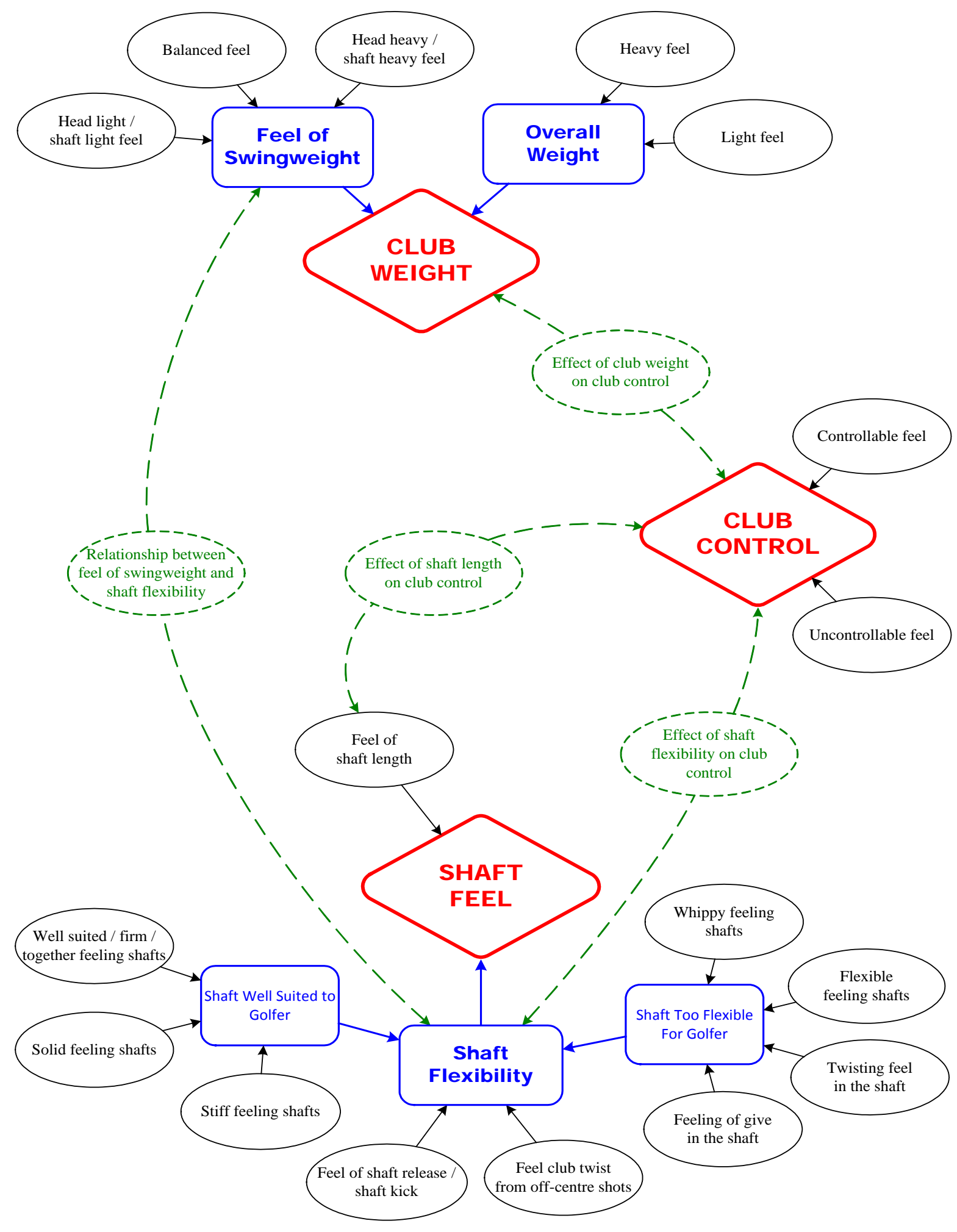

\section{KEY:}

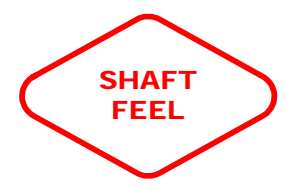

General Dimension

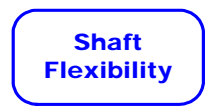

High Order Sub-Theme

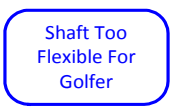

Low Order

Sub-Theme

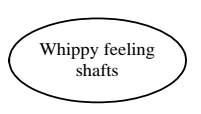

Base Level Theme

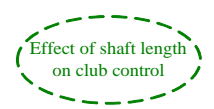

Inter-Dimension

Relationships

Figure 9 - Structured relationship model 
However, it cannot be said that the lighter a club is made the more controllable it becomes, there appears to be an optimum weight for each golfer.

That feels like I've lost the feel of swinging a weight... I don't want to feel something that's too heavy, but I've lost the feel of swinging a weight with that, that felt very light.

The final inter-dimension relationship illustrated in Figure 9 is between the flexibility of the shaft and the feel of swingweight.

I perceive that in a persimmon block, that the tip is almost too thin, which therefore makes this move a little bit more ... and that's what, for me, makes it (feel) a little bit heavier....

Golfers described feeling the head move more during the swing when a flexible shaft was installed in the club and this resulted in the perception of a heavier head.

\section{Discussion}

The purpose of this study was to elicit and understand golfers' perceptions of the equipment used when playing golf shots as previous studies had only made limited attempts to measure these subjective perceptions. This study has shown that manufacturers of golf products need to assess aspects of their equipment other than distance or power achieved. For example, a golfer needs to feel in control of the club and the subsequent flight of the ball, which, as the structured relationship modeling has shown, has direct implications for the weight of the club and the shaft fitted. Although only a relatively small number of golfers described these relationships, they suggest that such links exist. As Krane et al. (1997, p 215) state, "in many cases, rare experiences are no less meaningful, useful or important than common ones." Many golfers may have been unable to understand or explain, in this example, the source that results in a lack of control. It is also feasible that because the sample contains elite players, many of these golfers were able to adjust to variations in the equipment and able to control the many different clubs. The emergence of these relationships certainly warrants further investigation. However, not all the dimensions will be of equal importance and so further work is required to ascertain the relative importance of each dimension.

For example, the results of this qualitative study can also be used to develop instruments, such as questionnaires, with which data could more expediently be collected from a larger sample of elite golfers. Such an approach might allow investigators to quantify aspects of the emergent themes, such as their relative importance.

The approach used in this study can also be replicated to elicit perceptions of different golf equipment, of equipment used in other sports, and from alternative groups of performers. The procedure also highlights the success with which information can be gathered from performers during play, allowing experiences to be described as they arise and not in retrospect. In this study, the ability to interview the golfers whilst playing shots with a wide variety of clubs certainly increased the quantity and quality of information gathered. It can be argued that this principle should be applied to any player evalua- tion of equipment but this presents some difficulty with dynamic games such as tennis or squash compared with the quasi-static style of golf. The wireless recording system developed in this study could prove useful in allowing interviews to take place during the progress of a game.

Previous studies in sports psychology that have utilised the template of qualitative interviews followed by an inductive analysis did not consider interactions between the emergent dimensions. It is suggested that structured relationship modelling is one way of representing relationships when dimensions are not exclusive.

\section{Conclusions}

The aim of this study was to develop a formalised, qualitative approach for the elicitation of elite sports performers' perceptions of equipment used during play. An inductive analysis was performed on the raw data collected identifying core dimensions. Further analysis suggested inter relationships between the dimensions and a structured relationship model was created that enabled both the 'tree-structures' that emerged from the inductive analysis and the inter-dimension relationships to be illustrated. The authors suggest that the approach demonstrated in this study, using elite golfers and their perceptions of shots hit, can easily be adapted for different groups of players, alternative equipment or other sports.

\section{REFERENCES}

Biddle, S. J. H., Markland, D., Gilbourne, D., Chatzisarantis, N. L. D. and Sparkes, A. C. (200o). Research Methods in Sport and Exercise Psychology: Quantitative and Qualitative Issues. Journal of Sports Sciences (in press)

Cochran, A. and Stobbs, J. (1968). The Search For The Perfect Swing. London: Heinemann.

Cohen, L. and Manion, L. (1980). Research Methods in Education. London: Routledge.

Gould, D., Eklund, R. C. and Jackson, S. A. (1992a). 1988 U.S. Olympic Wrestling Excellence: 1. Mental Preparation, Precompetitive Cognition, and Affect. The Sport Psychologist, 6, 358-382.

Gould, D., Eklund, R. C. and Jackson, S. A. (1992b). 1988 U.S. Olympic Wrestling Excellence: 2. Thoughts and Affect Occurring During Competition. The Sport Psychologist, 6, 383-402.

Guba, E. G. (1978). Toward a Methodology of Naturalistic Inquiry in Educational Evaluation. CSE Monograph Series in Evaluation no. 8. Los Angeles: Center for the Study of Evaluation, University of California, Los Angeles.

Hanton, S. and Jones, G. (1999). The Acquisition and Development of Cognitive Skills and Strategies: I. Making the Butterflies Fly in Formation. The Sport Psychologist, 13, 1-21.

Harwood, C. G. (1997). Pre-Competition Achievement Goals Within Young Sports Performers. PhD Thesis, Loughborough University, Loughborough.

Hedrick, M. and Twigg, M. (1994). The Feel Of A Golf Shot: Can we measure it? In Golf: The Scientific Way (edited by Cochran, A.), pp. 131-133. Hemel Hempstead, U.K.: Aston Publishing Group.

Hocknell, A., Jones, R. and Rothberg, S. J. (1996). Engineering 'feel' in the design of golf clubs. In The Engineering of Sport: Proceedings of the ist International Conference on the Engineering of Sport (edited by Haake, S. J.), pp. 333-337. Rotterdam, NL: A.A.Balkema. 
Krane, V., Anderson, M. B. and Strean, W. B. (1997). Issues of Qualitative Research Methods and Presentation. Journal of Sport and Exercise Psychology, 19, 213-218.

Merkel, R. C. and Blough, T. (1999). Dynamic Characterisation and Comparison of Golf Clubs. In Proceedings of the 17th International Modal Analysis Conference pp. 513-517. FL, USA.

Merton, R. K. and Kendall, P. L. (1946). The Focused Interview. American Journal of Sociology, 51, 541-557.

Patton, M. Q. (1990). Qualitative Evaluation and Research Methods. London: Sage Publications.

Scanlan, T. K., Ravizza, K. and Stein, G. L. (1989a). An InDepth Study of Former Elite Figure Skaters: I. Introduction to the Project. Journal of Sport and Exercise Psychology, 11, 54-64.

Scanlan, T. K., Stein, G. L. and Ravizza, K. (1989b). An InDepth Study of Former Elite Figure Skaters: II. Sources of Enjoyment. Journal of Sport and Exercise Psychology, 11, 65-83.

Schmidt, R. A. (1991). Motor Learning and Performance: From Principles to Practice. Champaign, IL: Human Kinetics Books.

Sherwood, L. (1993). Human Physiology: From Cells to Systems. St. Paul, MN: West Publishing Company.

Varoto, P. S. and McConnell, K. G. (1995). Using Modal Analysis to Evaluate Golf Club Performance. Sound and Vibration, March 1995, 20-23. 